\title{
Numerical Solution of American Put Options Pricing with Transaction Cost in the CEV Model
}

\author{
Guojun Yuan ${ }^{1,2, a}$, Qingxian Xiao ${ }^{1, b}$ \\ ${ }^{1}$ Business School, University of Shanghai for Science and Technology, Shanghai, 200093, China \\ ${ }^{2}$ College of Economics and Management, West Anhui University, Lu'an, 237012, China \\ aemail:ygj1010@163.com, bemail:qxxiao@163.com
}

Keywords: Option Pricing; American Options; CEV Process; Transaction Cost; Semidiscretization

\begin{abstract}
In order to solve the American put options pricing and its numerical solution problems under the CEV model with transaction cost, by using the Itô formula and the no-arbitrage principle, the American put options pricing model and linear complementarity partial differential equation of the model are derived in this paper. Then the semi-discretization difference scheme for the American put options pricing model is developed, based on using semi-discretization for the spatial variable. Lastly, numerical experiments show that the semi-discretization difference scheme is a stable and convergent algorithm.
\end{abstract}

\section{Introduction}

Option is one of the most important financial derivative tools for corporations and investors over the past few decades. It has been used to keep way risk, hedge, and price financial assets etc.. Pricing options is the core problem in options theory. In 1973, famous financiers Fisher Black and Myron Scholes derived the famous option pricing model of European plain vanilla options (the B-S model)[1]. Most options traded around the world are American options which, unlike European options, can be exercised not just at expiry but at any time during the life of the option, many literatures have studied the American options pricing based on the B-S models assumptions [2-4].

One of hypothesis in the B-S model is that there isn't transaction cost in the transaction process, But in the actual finance market, there is transaction cost in the transaction process, the frequent transaction must be executed, for the asset's price changes perpetual. So investors have to face a mass of transaction cost, and the quantity of transaction cost is not ignorable [5]. It is not appropriate to apply the strategy of continuous trade with the transaction cost. Leland [6], Bensiad, Lesne and Pagés [7] studied the option pricing in the discrete condition. Leland [6]derived the differential equation of the option pricing model which was analogous to the differential equation of the B-S model by applying the $\delta$-hedging methods. In this paper, we consider the transaction cost caused by changing the proportion and develop the trade model in discrete time, in order to avoid the higher cost caused by the strategy of continuous trade, And derive the American put options pricing model and linear complementarity partial differential equation under the CEV model with transaction cost, by using Ito formula and the no-arbitrage principle. Then we also study the numerical solution of pricing American put option with transaction cost under the CEV model.

\section{The Model for pricing American put option with transaction cost in the CEV Process}

Another hypothesis in the B-S model is that the process of the price of asset is a geometric Brownian motion, for the present, mostly papers that study the American option pricing base on the hypothesis. However, the study in the literature[8], by studying the actual dates, found that the Brownian motion didn't accord to the actual condition. CEV is the abbreviation of 'Constant Elasticity of Variance', it means that elasticity of variance is a constant, introduced firstly by Cox and Ross[9], CEV has the characteristic that the asset price fluctuate is associate with the price level, and Cox[10]found that the 'variance smile' is caused by the negative correlation between the variance and the stock price fluctuate. 
Let $S(t)$ be a price process for a risk asset, in a given probability space $(\Omega, F, P)$, it satisfies the following stochastic differential equation:

$d S(t)=r S d t+\sigma S^{\alpha} d W(t)$

where $r$ is the riskless interest rate, $\sigma$ is the variance of the yield of asset , $\sigma>0, r, \sigma$ are constants. $W(t)$ is a standard Brownian motion, $\alpha$ is a constant, $0<\alpha \leq 1$, called elasticity gene.

Transaction cost is caused by the investor changing the proportion, usually the seller and the buyer pay it. Let $k$ denotes the transaction cost of one dollar trade, then the transaction cost is $k|\beta| S$ / 2 when buy or sell $\beta$ unit asset at the price $S$.

Considering the portfolio: buying an option and selling $\Delta$ quantity risk asset, then

$\Pi=\Delta S+B$

Where $\Delta$ denotes the quantity of the asset whose price is $S, B$ denotes riskless bond. Let $v$ denote the option value copied by the portfolio, and $v$ is the function of $S, t$, viz.: $v=v(S, t)$, by risk neutrality[1], we have $\Delta=\frac{\partial v}{\partial S}, B=v-\frac{\partial v}{\partial S} S$

Changing $\Pi$ in the trade of discrete time, During the time $\delta t$, the asset's price changes as:

$\delta S(t)=r S \delta t+\sigma^{\alpha} \delta W(t)$

When there is the transaction cost in the trade process, During the time of $\delta$, we have

$\delta \Pi=\Delta \delta S+r B \delta t-\frac{k}{2}|\delta \omega| S$

where $\delta S$ is the change of asset's price, $\delta \omega$ is the change quantity of the asset in the portfolio.

Based on (3), (4) then

$\delta \Pi=\sigma^{\alpha} \frac{\partial v}{\partial S} \delta W(t)+r v \delta t-\frac{k}{2}|\delta \omega| S$

During the time $\delta$, because of $\delta t \nrightarrow 0$, the change of the $\Delta$ gene can't be ignorable, its changing quantity is: $\Delta=\frac{\partial v}{\partial S}(S+\delta S, t+\delta t), \delta \omega=\frac{\partial v}{\partial S}(S+\delta S, t+\delta t)-\frac{\partial v}{\partial S}(S, t)$, by Ito's Lemma, we have $\frac{k}{2}|\delta \omega| S \approx$ $\frac{\sigma^{2}}{2} L_{e S} S^{1+\alpha}\left|\frac{\partial^{2} v}{\partial S^{2}}\right| \delta t$, where $L e=\sqrt{\frac{2}{\pi}}\left(\frac{k}{\sigma \sqrt{\delta t}}\right)$ is called the Leland number[6].

And so

$\delta \Pi=\sigma^{\alpha} \frac{\partial v}{\partial S} \delta W(t)+r v \delta t-\frac{1}{2} \sigma^{2} L e S^{1+\alpha}\left|\frac{\partial^{2} v}{\partial S^{2}}\right| \delta t$

Considering $v=v(S, t)$, by using the Itô formula [11], the no-arbitrage principle, we have

$\frac{\partial v}{\partial t}+r S \frac{\partial v}{\partial S}+\frac{1}{2} \sigma^{2}\left(S^{2 \alpha} \frac{\partial^{2} v}{\partial S^{2}}+L e S^{1+\alpha}\left|\frac{\partial^{2} v}{\partial S^{2}}\right|\right)-r v=0$

Let $\tau=T-t, \quad u(S, \tau)=v(S, t)$, reversal time question (7) becomes the following forward time question:

$$
\frac{\partial u}{\partial \tau}-r S \frac{\partial u}{\partial S}-\frac{1}{2} \sigma^{2}\left(S^{2 \alpha} \frac{\partial^{2} v}{\partial S^{2}}+L e S^{1+\alpha}\left|\frac{\partial^{2} v}{\partial S^{2}}\right|\right)+r v=0
$$

American options can be exercised at any time before expiry. Due to this case, an additional constraint has to be introduced to the model to avoid arbitrage opportunities. Thus, the value $u$ of an American put option can be obtained by solving an linear complementarity partial differential equation:

$$
\begin{aligned}
& \left(\frac{\partial u}{\partial \tau}-r S \frac{\partial u}{\partial S}-\frac{1}{2} \sigma^{2} S^{2 \alpha} \frac{\partial^{2} u}{\partial S^{2}}-\frac{1}{2} \sigma^{2} L e S^{1+\alpha} \frac{\partial^{2} u}{\partial S^{2}}+r u\right) \times(u-g)=0 \\
& \frac{\partial u}{\partial \tau}-r S \frac{\partial u}{\partial S}-\frac{1}{2} \sigma^{2}\left(S^{2 \alpha}+L e S^{1+\alpha}\right) \frac{\partial^{2} u}{\partial S^{2}}+r u \leq 0 \\
& g=g(S)=(K-S)^{+} \\
& u(\tau, 0)=K, u(T, S)=\left(K-S_{T}\right)^{+}, \lim _{S \rightarrow+\infty} u(\tau, S)=0
\end{aligned}
$$




\section{Semi-discretization difference scheme of the linear complementarity partial differential equation}

In order to construct the computing difference scheme, we truncate the infinite domain at $S=X$ with $X$ being sufficiently large and pose boundary condition $u(\tau, X)=0$. To avoid arbitrage opportunities, the American option prices has to satisfy an early exercise constraint $u(\tau, S) \geq G(S)$ for all $(\tau, S) \in[0, T] \times[0, X]$.

Let $h=\Delta S=S_{i}-S_{i-1}=X / N, i=1,2, \cdots, M$, the space derivatives of Ineq.(10) are approximated with central-differences $\frac{\partial u}{\partial S}\left(S_{i}, \tau\right) \approx \frac{u\left(S_{i+1}, \tau\right)-u\left(S_{i-1}, \tau\right)}{2 h} \hat{=} \nabla_{i}(\tau), \frac{\partial^{2} u}{\partial S^{2}}\left(S_{i}, \tau\right) \approx \frac{u\left(S_{i+1}, \tau\right)-2 u\left(S_{i}, \tau\right)+u\left(S_{i-1}, \tau\right)}{2 h^{2}} \hat{=} \Delta_{i}(\tau)$.

Let $U_{i}(\tau)$ be the approximation of theoretical value $u\left(S_{i}, \tau\right)$, and $U(\tau)=\left[U_{1}, U_{2}, \cdots, U_{M-1}\right]^{T}$. Boundary values $U_{0}, U_{M}$ appearing are computed by using second-order Lagrange interpolating polynomial passing through the two closest internal mesh points,that is: $U_{0}=2 U_{1}-U_{2}, U_{M}=2 U_{M-1}-U_{M-2}$. Then gets the semidiscretization approximating problem form as following for the partial differential inequation (10):

$$
\left\{\begin{array}{l}
\frac{d U(\tau)}{d \tau} \leq C U(\tau), 0 \leq \tau \leq T \\
U(0)=\left[U_{1}(0), U_{2}(0), \cdots, U_{M-1}(0)\right]^{T}, U_{i}(0)=\left(K-S_{i}\right)^{+}
\end{array}\right.
$$

where $\quad C=\left(\begin{array}{cccccc}a_{1}+2 c_{1} & b_{1}-c_{1} & 0 & 0 & \cdots & 0 \\ c_{2} & a_{2} & b_{2} & 0 & \cdots & 0 \\ 0 & c_{3} & a_{3} & b_{3} & \cdots & 0 \\ \vdots & \vdots & \ddots & \ddots & \ddots & \vdots \\ 0 & \cdots & 0 & c_{M-2} & a_{M-2} & b_{M-2} \\ 0 & \cdots & 0 & 0 & c_{M-1}-b_{M-1} & a_{M-1}+2 b_{M-1}\end{array}\right)$

$a_{i}=-\frac{\sigma^{2} S_{i}^{2 \alpha}+\sigma^{2} L e S_{i}^{1+\alpha}+r h^{2}}{h^{2}}, b_{i}=-\frac{\sigma^{2} S_{i}^{2 \alpha}+\sigma^{2} L e S_{i}^{1+\alpha}+r h S_{i}}{2 h^{2}}, \quad c_{i}=-\frac{\sigma^{2} S_{i}^{2 \alpha}+\sigma^{2} L e S_{i}^{1+\alpha}-r h S_{i}}{2 h^{2}}, \quad i=1, \cdots, M-1$.

Using Euler method

$$
U((n+1) \Delta \tau) \leq(I+C) U(n \Delta \tau), 0 \leq n \leq N
$$

Where $I$ is $N-1$ order unit matrix, $\Delta \tau=T / N$.

For American put options, we obtain a semi-discrete linear complementarity problem

$$
\left\{\begin{array}{l}
U(\tau)-\left[\prod_{n=0}^{N-1}(I+\Delta \tau C)\right] U(0) \leq 0, \quad U \geq G \\
\left(U(\tau)-\left[\prod_{n=0}^{N-1}(I+\Delta \tau C)\right] U(0)\right)(U-G)=0
\end{array}\right.
$$

\section{Numerical experiments}

Consider the American put options with the following parameter: $S_{0}=45, r=0.1, \sigma=0.2, K=50$, $X=200$.

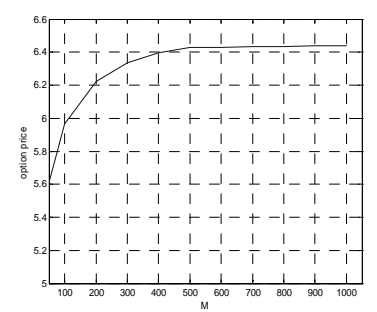

(a) the relation between $M$ and option price

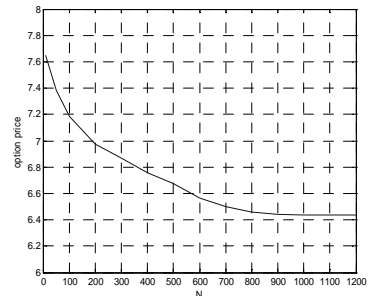

(b) the relationship between $N$ and option price

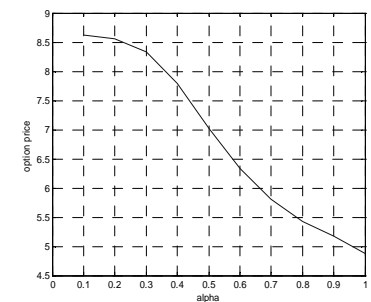

(c) the relationship between $\alpha$ and option price

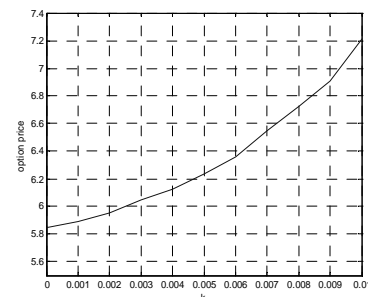

(d) the relationship between $k$ and option price

Fig.1. The experimental results 
Figure 1(a). shows the relation between space step number $M$ and option price, it can be found that the option price is convergent when $M$ value increases. Figure 1(b). shows the relation between time step number $N$ and option price, it can be found that the option price is convergent when $N$ value increases. Figure 1(c). shows the relation between $\alpha$ and option price, it can be found that the option price decreases when $\alpha$ value increases. Figure $1(\mathrm{~d})$. shows the relation between proportion of transaction cost $\mathrm{k}$ and option price, it can be found that the option price increases when $k$ value increases, it is consistent with the actual finance market.

\section{Conclusion}

The paper mostly studies a new numerical method for pricing American put options with transaction cost under the CEV model, and derives the differential equation of the option pricing model by using the Itô formula and the no-arbitrage principle. Then we develop the semi-discrete.

\section{Acknowledgement}

This work was supported by the NNSF of China (Project No. 11171221) and Shanghai Leading Academic Discipline Project(Project No.XTKX2012).

\section{References}

[1] Fisher Black, Myron Scholes. The pricing of options and corporate liabilities [J]. Journal of Political Economy. 1973:81(3):637- 655.

[2] Giovanni Barone-Adesi, Robert E. Whaley. Efficient analytic approximation of American option values [J] Journal of Finance.1987:42(2):301- 320.

[3] Yue-Kun Kwok. Mathematical Models of Financial Derivatives [M]. Berlin: Springer-Verlag, 1998. 229- 251.

[4] Samuli Ikonen, Jari Toivanen. Pricing American options using LU decomposition [J]. Appl. Math. Sci. 2007:1(51):2529- 2551.

[5] Guy Barles, Halil Mete Soner. Option pricing with transaction costs and a nonlinear Black-Scholes equation [J]. Finance Stochast.1998:2(4):369-397.

[6] Hayne E. Leland. Option pricing and replication with transaction cost [J]. J. Finance, 1985:40(5):1283- 1301.

[7] Bernard Bensaid, Jean-Philippe Lesne, Henri Pagés, José Scheinkman. Derivative asset pricing with transaction costs [J]. Math. Finance. 1992:2(2):63- 86.

[8] Andrew W. Lo, A. Craig Mackinalary. Stock market prices do not follow random wolks:evidence from s simple specification test [J]. Review of Financial Studies. 1988:1(1):41-66.

[9] Cox John C., Ross Stephen A. The valuation of option for alternative stochastic process [J]. Journal of Financial Economics. 1976:3(1-2):145-166.

[10] Cox John C. The constant elasticity of variance option pricing model [J]. Journal of Portfolio Management. 1996 (23) 15- 17.

[11] Peter Tankov, Rama Cont. Financial modeling with jump process [M]. Boca Raton: Chapman and Hall/CR, 2004.241-276. 